\title{
Risk indicators of reduction limb defects
}

\author{
T ARO, ${ }^{1}$ O P HEINONEN, ${ }^{2}$ AND L SAXÉN ${ }^{1}$ \\ From the Department of Pathology, ${ }^{1}$ University of Helsinki, and the National Public Health Institute, ${ }^{2}$ Helsinki, \\ Finland
}

SUMMARY The birth of a child with a reduction limb defect (RLD) was evaluated in relation to vaginal bleeding, threatened abortion, and other complications of pregnancy, placental weight, birth weight, family history, parental age, and the outcome of previous pregnancies. The material consisted of 453 cases of reduction limb defect and an equal number of non-malformed controls matched for time and place. The children were born in Finland during 1964-77. The cases with reduction limb defect without additional malformations were analysed separately. Statistically significant associations were found between the occurrence of reduction limb defect and the following risk indicators: vaginal bleeding, threatened abortion, duration of gestation under 37 weeks, placental weight $400 \mathrm{~g}$ or less, birth weight $2500 \mathrm{~g}$ or less, and any type of malformation in the relatives., Vaginal bleeding indicated the risk of reduction limb defect to be increased about fourfold; short gestation indicated about twofold risk of reduction limb defect as an isolated malformation. Both low placental weight and low birth weight were associated to a threefold risk of an isolated reduction limb defect. These factors of an abnormal pregnancy indicated even higher risk of reduction limb defect with additional malformations. Preliminary genetic analysis suggests that hereditary factors play no major part in the aetiology of reduction limb defects.

The first reports on thalidomide as a cause of human limb malformation appeared in $1961 . .^{12}$ Despite the active research triggered by these reports, little is known about the aetiology of reduction limb defects in man. In addition to thalidomide, only tetracyclines $^{34}$ and folic acid antagonists ${ }^{5}$ are suggested as constituting a teratogenic risk to the developing skeletal system. The use of anticonvulsants ${ }^{6}$ and oral contraceptives ${ }^{7}$ during pregnancy has also been reported to increase the risk, but other studies fail to support these findings..$^{8-10}$

Congenital defects are associated with several infections, but hypoplasia or reduction of the skeletal structures of the limbs has been reported to occur in excess only in connection with herpes simplex, ${ }^{11}$ varicella, and herpes zoster. ${ }^{12} 13$

In the collaborative perinatal project ${ }^{14}$ advanced maternal age (over 40 years), low birth weight of the child (under $2000 \mathrm{~g}$ ), and low placental weight (under $300 \mathrm{~g}$ ) were related to an increased risk of a child's musculoskeletal malformation. In another report reduction limb defect was associated with young parental age and maternal toxaemia. ${ }^{15}$

In this study some less frequently evaluated factors are examined in relation to the occurrence of reduction limb defect-namely, the outcome of previous pregnancies, an abnormal course of the pregnancy, and the family history. Several statistically significant associations were found.

\section{Material and methods}

The material consists of 453 cases of reduction limb defect collected by the Finnish Registry of Congenital Malformations during 1964-77. In this period a total of 964.878 children over $600 \mathrm{~g}$, including stillbirths, were born in Finland.

The registry is a national surveillance system with data based on reports of all congenital malformations detected in children under 1 year of age in Finland. ${ }^{16}$ Skeletal defects were selected to be studied in detail. Each case selected for the study was matched with a control for time and place. The birth of the control immediately preceded the birth of the case in the same maternity welfare district. There were no incomplete pairs.

Cases of reduction limb defect that were part of a known syndrome with recognisable genetic background were excluded ( 3 cases). The remaining cases were confirmed by radiography (378 cases), necropsy records (43), or clinical records only (32). In 314 of the children only one or both arms were 
affected, in 90 only legs, and in 49 both arms and legs. The deficiencies have been described in detail. ${ }^{17}$

The total study group of the reduction limb defects (TRLD) included cases with additional defects of various organs. Thus two subgroups were formed: isolated reduction limb defects with no additional malformations (IRLD, 329 cases) and associated reduction limb defects (ARLD, 124 cases).

The mothers of the children with these defects were interviewed by midwives. Using structured questionnaires they sought information on previous pregnancies, the recent pregnancy, and the family. Records of the particular maternity welfare centres ${ }^{18}$ that the mothers had visited since early pregnancy were also perused. Thus the information of the events during the pregnancy had been registered before the birth of the malformed child. The questionnaires were completed and other records used for mothers of the controls in an identical way.

The mothers' replies concerning defects in the relatives of the cases were compared with the replies of the mothers of the controls. Often, however, it was not possible to figure out the exact number of relatives. Also two other groups of skeletal defects-namely, polydactyly (559 cases) and syndactyly (299)-both with a known heritable trend, were studied for a familial factor. These cases have been described.17

To measure association between a factor and a defect the statistic "relative risk" was used. A relative risk is the ratio of the rate of a defect in the case group to the rate in the control group. Estimates of relative risks here are relative odds (RO) that have been presented with $95 \%$ confidence intervals ( $\left.\mathrm{CI}_{95}\right)$. Relative odds and confidence intervals for matched pairs were computed by the method of Breslow et al. ${ }^{19}$

\section{Results}

VAGINAL BLEEDING AND THREATENED ABORTION

To analyse the effect of vaginal bleeding, the mothers were divided into two groups-those who had vaginal bleeding during pregnancy and those who had not. Among the case-control pairs, the mother of the child with a limb reduction defect had more often had vaginal bleeding than the control mother of the non-malformed child, both during the first trimester of pregnancy and later. Taking the control mothers with no vaginal bleeding as referents, the relative odds for mothers with vaginal bleeding was statistically significantly greater in the total study group (TRLD) and in the subgroup of isolated reduction limb defects (IRLD) (table 1). The tendency was the same in the subgroup of associated
Table 1 Vaginal bleeding during pregnancy and reduction limb defects. (Matched pairs of total study group without parentheses, pairs of isolated reduction limb defects in parentheses)

\begin{tabular}{lcc}
\hline & \multicolumn{2}{l}{ Cases } \\
\cline { 2 - 3 } Controls & Bleeding & No bleeding \\
\hline I trimester & $0(0)$ & $10(9)$ \\
Bleeding & $39(31)$ & $378(209)$ \\
No bleeding & $3 \cdot 9(3 \cdot 4)$ & $1 \cdot 0^{*}(1 \cdot 0)^{*}$ \\
Relative odds & $2 \cdot 1-7 \cdot 4$ & \\
95\% confidence interval & $(1 \cdot 7-6 \cdot 9)$ & \\
& $17(0)$ & $2(2)$ \\
II-III trimester & $17(10)$ & $378(209)$ \\
Bleeding & $8 \cdot 5(5 \cdot 0)$ & $1 \cdot 0^{*}(1 \cdot 0)^{*}$ \\
No bleeding & $2 \cdot 5-28 \cdot 8$ & \\
Relative odds & $(1 \cdot 3-19 \cdot 6)$ & \\
95\% confidence interval & & \\
\hline
\end{tabular}

*Reference category.

Table 2 Number of discordant matched pairs (cases of reduction limb defects/controls) with threatened abortions

\begin{tabular}{lll}
\hline & Threatened abortion \\
\cline { 2 - 3 } \cline { 2 - 3 } & I trimester & II-HI trimester \\
\hline Total study group & $7 / 0$ & $4 / 1$ \\
Isolated limb reduction defects & $6 / 0$ & $0 / 0$ \\
Associated limb reduction defects & $1 / 0$ & $4 / 0$ \\
\hline
\end{tabular}

reduction limb defects (ARLD), but the number of discordant pairs was small (I trim RO 8/1, II-III trim $7 / 0)$.

Threatened abortion (bleeding and pain) during the first trimester was associated with the TRLD and the IRLD. The associations were statistically significant. Threatened abortion during the second or third trimester was most frequent among the mothers of the TRLD and the ARLD cases (table 2).

To analyse the relationship of reduction limb defect to vaginal bleeding or threatened abortion, or both, during the first trimester (1-14 weeks of gestation), the exact stage of gestation was determined (fig 1). Of all the 39 cases, in six vaginal bleeding or threatened abortion, or both, occurred before "the sensitive period" of thalidomide-type limb reduction defects, in 17 within the sensitive period, and in 16 after the sensitive period. Most of the later cases (9/7) had major defects or multiple malformations. In 24 cases out of 39 the gestation ended before the 39th week.

DURATION OF GESTATION, PLACENTAL WEIGHT, BIRTH WEIGHT, AND SURVIVAL OF THE CHILD The duration of the gestation was statistically significantly shorter in the mothers of the TRLD and the IRLD cases (table 3 ). A short gestation was even more clearly associated with the ARLD (gestation of 


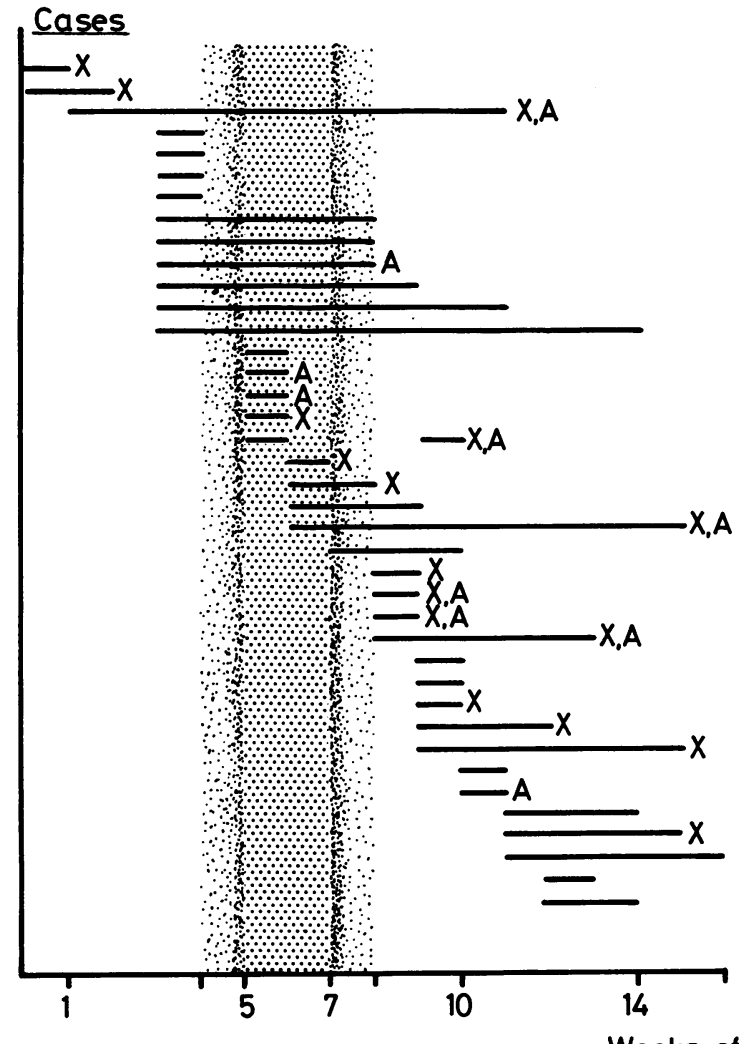

Weeks of gestation

Table 3 Duration of pregnancy and reduction limb defects. (Matched pairs of total study group without parentheses, pairs of isolated reduction limb defects in parentheses)

\begin{tabular}{|c|c|c|c|}
\hline \multirow[b]{2}{*}{ Controls } & \multicolumn{3}{|l|}{ Cases } \\
\hline & $<37$ weeks & 37-39 weeks & $>39$ weeks \\
\hline$<37$ weeks & $7 \quad(4)$ & $6(5)$ & $21 \quad(20)$ \\
\hline 37-39 weeks & 23 (12) & 20 (15) & $55 \quad(48)$ \\
\hline$>39$ weeks & $75 \quad(39)$ & $59 \quad(38)$ & 178 (141) \\
\hline Relative odds & $3.7(1.9)$ & $1 \cdot 1 \quad(0 \cdot 8)$ & $1.0^{*}(1.0)^{*}$ \\
\hline interval & $\begin{array}{c}2 \cdot 5-6 \cdot 1 \\
(1 \cdot 2-3 \cdot 3)\end{array}$ & $\begin{array}{c}0 \cdot 8-1 \cdot 5 \\
(0 \cdot 5-1 \cdot 2)\end{array}$ & $\overline{(-)}$ \\
\hline
\end{tabular}

*Reference category.

Table 4 Placental weight and reduction limb defects. (Matched pairs of total study group without parentheses, pairs of isolated reduction limb defects in parentheses)

\begin{tabular}{lcl}
\hline & \multicolumn{2}{l}{ Cases } \\
\cline { 2 - 3 } Controls & $\leq 400 \mathrm{~g}$ & $>400 \mathrm{~g}$ \\
\hline$\leq 400 \mathrm{~g}$ & $1 \mathrm{(0)}$ & $11(10)$ \\
$>400 \mathrm{~g}$ & $78(35)$ & $302(319)$ \\
Relative od & $7 \cdot 1(3 \cdot 5)$ & $1 \cdot 0^{*}(1 \cdot 0)^{*}$ \\
$95 \%$ confidence interval & $4 \cdot 1-12 \cdot 2)$ & - \\
& $(1 \cdot 8-6.8)$ & $(-)$ \\
\hline
\end{tabular}

*Reference category.
Fig 1 Vaginal bleeding and threatened abortion by week of gestation. $X=$ major $\operatorname{limb}$ defect, $A=$ associated malformations, hatched bar = sensitive period for thalidomide. ${ }^{22}$

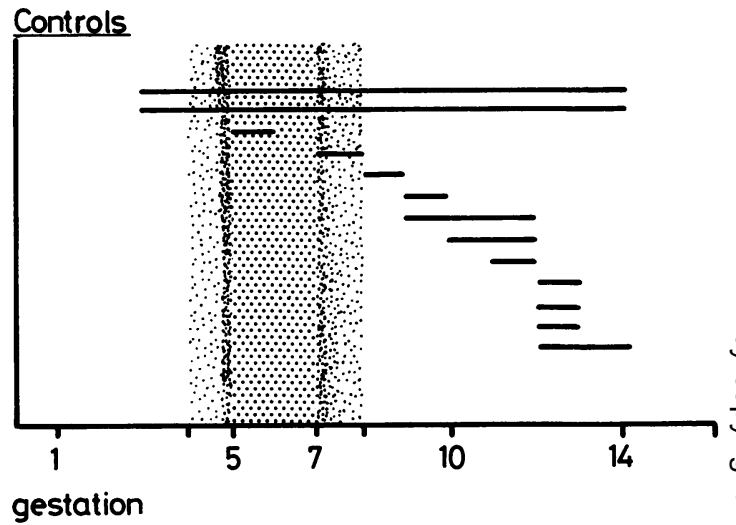

37-39 weeks: RO 3.0, CI9s 1.5-9.5; gestation of 37 weeks or under: RO $35 \cdot 0, \mathrm{CI}_{95} 13 \cdot 7-83 \cdot 0$ ).

Low placental weight was associated with defects in the TRLD and in the subgroup of the IRLD (table 4). Again, this was most clearly observed in the ARLD subgroup (RO 43.0, CI95 13.4-137.8).

In the TRLD and in the IRLD groups low birth weight was associated with the birth of a defective child (table 5). The highest relative odds for the birth weight of $2500 \mathrm{~g}$ or less was observed in the ARLD subgroup (RO 30.0, CI9s 12.3-86.8).

Table 5 Birth weight and reduction limb defects. (Matched pairs of total study group without parentheses, pairs of isolated reduction limb defects in parentheses)

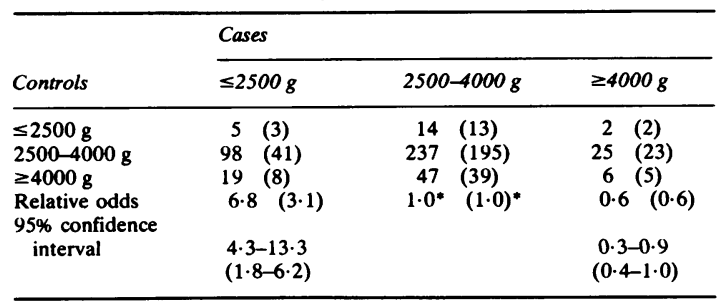

${ }^{*}$ Reference category. 
Table 6 Survival of the child and reduction limb defects. (Matched pairs of original study group without parentheses, pairs of isolated reduction limb defects in parentheses)

\begin{tabular}{lll}
\hline & \multicolumn{2}{l}{ Cases } \\
\cline { 2 - 3 } Controls & $\begin{array}{l}\text { Stillborn or died } \\
\text { afier birth }\end{array}$ & Alive \\
\hline Stillborn or died after birth & $0(0)$ & $5(5)$ \\
Alive & $75(12)$ & $369(310)$ \\
Relative odds & $15 \cdot 0(2 \cdot 4)$ & $1 \cdot 0^{*}(1 \cdot 0)^{*}$ \\
$95 \%$ confidence interval & $7 \cdot 6-30 \cdot 0$ & - \\
& $(0 \cdot 9-6 \cdot 6)$ & $(-)$ \\
\hline
\end{tabular}

* Reference category.

The number of children who were born dead or died soon after birth was much higher in the case group than in the control group. No statistically significant difference was noted in the subgroup of the IRLD (table 6). In the ARLD group the proportion of non-survivors was increased most (RO $63 / 0)$.

REDUCTION LIMB DEFECTS AND OTHER MALFORMATIONS IN THE RELATIVES

In the TRLD and the IRLD subgroups there were significantly more malformations in the family than in the families of the controls (RO 2.5, CI9s 1.7-3.5; RO 2.6, CI 95 1.8-3.9). In the smaller ARLD subgroup the relative odds were of the same magnitude, but it was not statistically significant (RO $\left.2 \cdot 0 \mathrm{CI}_{95} 1 \cdot 0-4 \cdot 2\right)$.

The mothers were also interviewed for specific defects known in the close relatives (siblings of the affected child, parents, their siblings, or grandparents). Distribution of three types of limb malformations and "other defects" in the relatives of the TRLD is shown in fig 2. For comparison, identically collected data from the families of the controls, the polydactylous children, and the syndactylous propositi are presented. A stronger and more specifically familial trend is seen in the two latter groups, whereas the number of deformities in the pedigree of the study group (TRLD) is small and the distribution by type seems random.

The children with reduction defects had 19 relatives with the same type of deformity, whereas children in the matched pair control group had only five near relatives with reduction defects. An attributable risk of a history of reduction limb defect in relatives is about $3 \%$ among children with this defect.
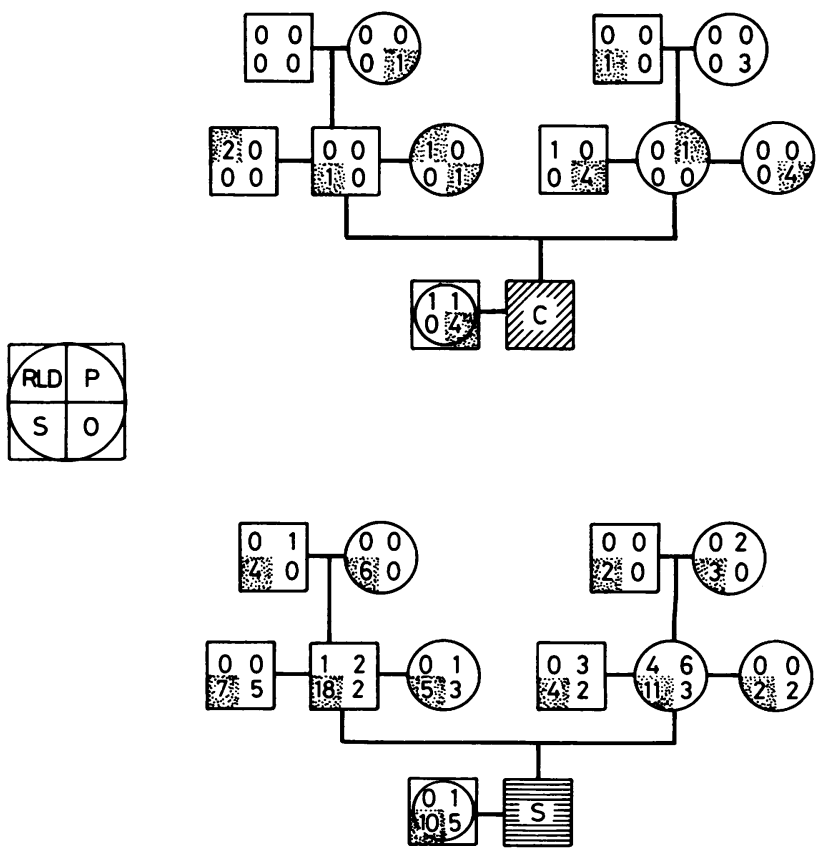

Fig 2 Modified pedigree charts of relatives of 453 children with reduction limb deformity (RLD), of 453 control children $(C)$ matched for time and place, of 559 children with polydactyly $(P)$, and of 299 children with syndactyly $(P)$, and of 299 children with syndactyly (S). All siblings of the propositi and all siblings of their parents are presented by one circle and one square. Sisters and brothers of the propositi are presented in the same "circle-square." Four numbers starting clockwise from upper left quadrate mean number of affected individuals with $R L D, P$, and other malformations (O), and $S$. The largest number in each circle or square has been darkened. 
The cases had other malformations in 39 relatives and the controls in 21 relatives, showing a risk of about $4 \%$ attributable to a history of any other malformation in the relatives. In contrast, the attributable risk for a family history of syndactyly was about $25 \%$ among the cases of syndactyly. It was about $9 \%$ for a family history of any other malformation. Among polydactylous children the attributable risks were $24 \%$ and $1 \%$, respectively.

If a mother had previously had a malformed child the relative odds were significantly increased (RO $\left.2 \cdot 8, \mathrm{CI}_{95} 1 \cdot 1-7 \cdot 4\right)$ in the TRLD. In both subgroups the risk was also increased but the confidence intervals included the unity (IRLD RO 2.5, CI9s 0.8-7.7; ARLD RO 4.0, CI 95 0.5-30.0).

Table 7 summarises the statistically significant associations.
NON-SIGNIFICANT ASSOCIATIONS

Parental age, marital state, previous live births, birth order, previous stillbirths, and previous abortions were not statistically significantly associated with reduction limb defect (table 8 ).

\section{Discussion}

This study has limitations and potential errors. Not all of the reduction limb defects are reported to the Finnish Registry of Congenital Malformations. The study material suffers from underreporting of about $30 \% .20$ Particularly the minor defects are underreported. ${ }^{17}$ Thus the observed associations might be stronger than in a totally unselected series. This should not, however, materially affect the conclusions.

Table 7 Summary of the relative odds for the risk indicators statistically significantly associated with the occurrence of a reduction limb defect in a time and place matched case-control series of children born in 1964-77 in Finland

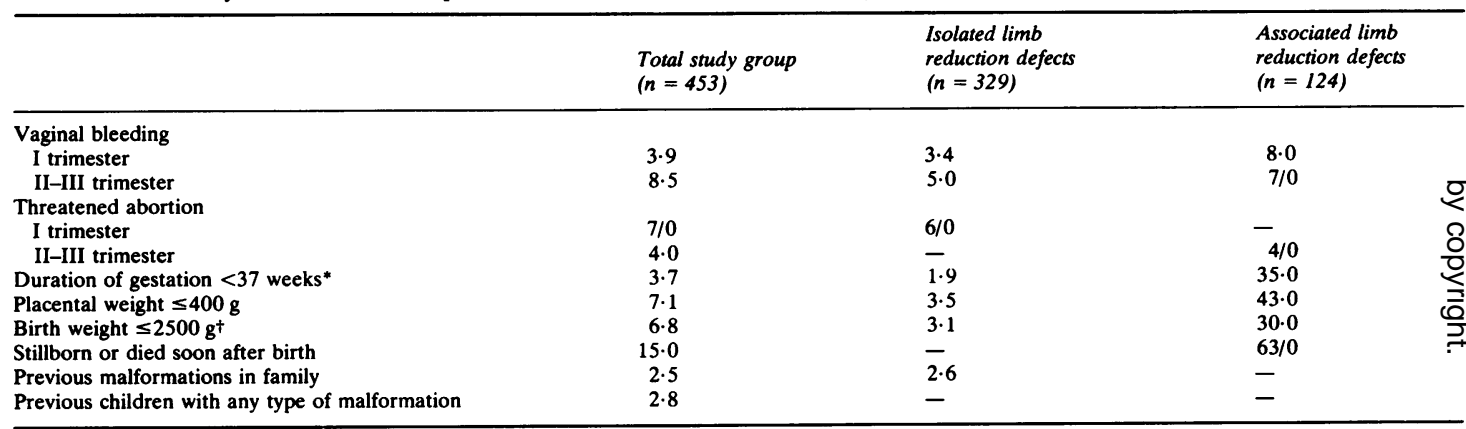

*Reference category $>39$ weeks.

$\dagger$ Reference category $2500-4000 \mathrm{~g}$.

Table 8 Relative odds for factors showing statistically non-significant associations with the occurrence of reduction limb defect in a time and place matched case-control series of children born in 1964-77 in Finland

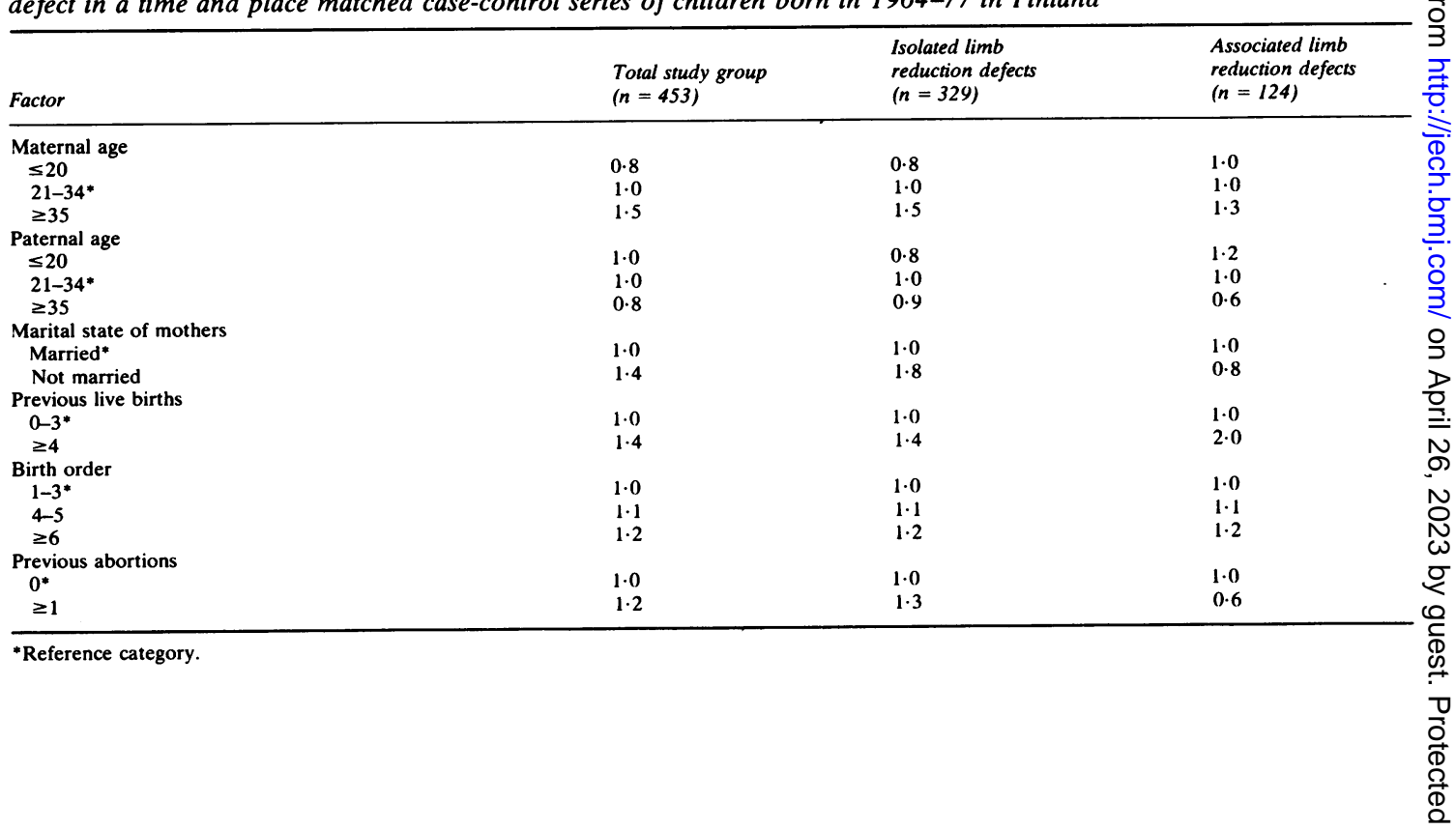


Some of the data are from the antenatal records of the maternity welfare centres, but retrospective information was also obtained in the interviews. Hence, the bias of maternal memory could affect some of the findings.

The association between a variable and a defect in this report may also be false because of the presence of another covariate-that is, a factor showing a closely related matter, such as a mother's age and parity.

A confounding bias rises when some third factor has an effect on a study variable and the occurrence of the defect. The effect of an unknown confounding factor could have been partly reduced by using matched pair controls. Effect of covariants or confounding factors calls for multivariate analysis.

"Vaginal bleeding" and "threatened abortion" may reflect an effort to expel the embryo. This condition was significantly associated with a raised risk of subsequently giving birth to a child with reduction limb defect without or, especially, with, associated malformations. Four alternative explanations for the association can be offered: either we are dealing with a maternal memory bias, ${ }^{21}$ a true aetiological factor, a consequence of the abnormal state of the embryo, or an unknown factor is independently causing both malformation and abortion. The explanation by a selective memory bias does not apply here because the information about bleeding and signs of a threatened abortion was derived from the prospective records of the antenatal clinics. Yet the other three alternatives remain, and their analysis meets great difficulties. An effort was made to distinguish between a common cause and a consequence by exploring the stage of pregnancy during bleeding, the assumed phase of skeletogenesis, and also the severity of the defect (fig 1).

Owing to retrospective analyses of the thalidomide disaster, some information is available on the sensitive period of the reduction limb defects in human embryos. Accordingly, the sixth and seventh weeks of pregnancy seem to be the most vulnerable periods. ${ }^{22}$ Embryologically, this corresponds with the appearance of the limb buds and of the precartilagineous mesenchymal condensates of the long bones. Towards the end of this period the ossification centres appear. With a certain degree of caution one may conclude that insults during the sixth and seventh weeks of gestation could lead to reduction limb defects, after which actual bone formation might not be prevented, and exogeneous factors may merely impair growth and remodelling of the skeleton. If this contemplation is valid the 23 first cases in fig 1 (bleeding/threatened abortion before the seventh week of gestation) could reflect an insult manifested both as a threatened abortion and a reduction limb defect, whereas those of later stages can hardly be attributed to a common factor. They should reflect both real efforts to expel a severely defected embryo and merely coincidental phenomena; also the control mothers had occasional bleeding and threatened abortion. The incidence of defects is high among aborted embryos, thus supporting the idea of a natural elimination of defective embryos.

Whether or not vaginal bleeding and threatened abortion reflect a causal teratogenic factor, the fact remains that this disorder should be considered an important indicator for impaired embryogenesis. The conclusion is supported by two previous analyses of the material of the Finnish Registry of Congenital Malformations, where threatened abortion was shown to be associated with a raised risk of defects of the central nervous system and oral clefts. ${ }^{21}{ }^{23}$

Short gestation, low placental weight, and low birth weight were associated with an increased risk of all subgroups of reduction limb defect. Most strikingly, the association was in the subgroup of the ARLD, which implies the strong effect of associated malformations. There was also an association with the IRLD, which raises a question of the effect of a third factor. It is not easy to understand why a reduction limb defect would shorten the duration of gestation or have any influence on birth weight or placental weight.

Paternal malformations have been reported to be related to an increased risk of musculoskeletal malformations. ${ }^{14}$ Here the relation was to malformations in any near relative of the father or the mother.

In the present study a rough and tentative effort was made to examine defects in the near relatives of the affected children further. Only weak trends were detected in the relatives of the children with reduction defects, whereas such were quite obvious in a similar type distribution analysis of the other skeletal defects-syndactyly and polydactyly.

Ignoring the possible memory bias, assuming as many relatives of the cases as of the controls, and taking these rather small numbers for granted, an attributable risk of a history of reduction limb defect in siblings, parents, their siblings, or grandparents is negligible $(3 \%)$ among children born with a reduction limb defect. In contrast, any attributable risk of a history of a specific malformation among the cases of syndactyly and polydactyly was about eightfold.

A true genetic analysis of the heredity of reduction limb defects would require a proper family study, whereas these preliminary results suggest that type-specific hereditary factors might have some 
effect but play no major part in the aetiology of reduction limb defects.

In conclusion, several of the factors analysed in this study showed a statistically significant association with the occurrence of a reduction limb defect. Such risk indicators may prove useful in the future in delineating the maternal "risk groups" for intensive intrauterine surveillance. Furher indicators might be found by exploring maternal diseases and drug consumption when the analysis of this material continues.

We thank Mr Kimmo Ronkainen for his valuable help in computer programming. The study has been supported by the National Board of Health.

\section{References}

${ }^{1}$ McBride WG. Thalidomide and congenital abnormalities. Lancet 1961; ii: 1358.

${ }^{2}$ Lenz W. Kindliche Missbildungen nach Medikament-Einnahme während der Gravidität. Dtsch Med Wochenschr 1961; 86: 2555-6.

${ }^{3}$ Cohlan SQ, Bevelander G, Tiamsic T. Growth inhibition of prematures receiving tetracycline. Am J Dis Child 1963; 105: 453-61.

${ }^{4}$ Baker KL, Storey E. Tetracycline induced tooth changes. Part I-IV. Med J Aust 1970; 111: 109-13, 114-9.

${ }^{5}$ Warkany J. Aminopterin and methotrexate: folic acid deficiency. Teratology 1978; 17: 353-8.

${ }^{6}$ Loughnan PM, Gold H, Vance JC. Phenytoin teratogenicity in man. Lancet 1973; i: 70-2.

${ }^{7}$ Janerich DT, Piper JM, Glebatis DM. Oral contraceptives and congenital limb-reduction defects. $N$ Engl J Med 1974; 291: 697-700.

${ }^{8}$ Shapiro S, Hartz SC, Siskind V, et al. Anticonvulsants and parental epilepsy in the development of birth defects. Lancet 1976; i: 272-5.
${ }^{y}$ David TC, O'Callaghan M. Birth defects and oral hormone preparations. Lancet 1974; i: 1236.

${ }^{10}$ Savolainen E, Saksela E, Saxén L. Teratogenic hazards of oral contraceptives analyzed in a national malformation register. Am J Obstet Gynecol 1981; 140: 521-4.

${ }^{11}$ Montgomery JR, Flanders RW, Yow MD. Congenital anomalies and herpesvirus infection. Am J Dis Child 1973; 126: 364-6.

${ }^{12}$ Savage MO, Moosa A, Gordon RR. Maternal varicella infection as a cause of fetal malformations. Lancet 1973; i: $352-4$.

${ }^{13}$ De Nicola LK, Hanshaw JB. Congenital and neonatal varicella. J Pediatr 1979; 94: 175-6.

${ }^{14}$ Heinonen OP, Slone D, Shapiro S. Birth defects and drugs in pregnancy. Littleton, Maryland: Publishing Sciences Group Inc, 1977.

${ }^{15}$ Rogala EJ, Wynne-Davies R, Littlejohn A, Gormley J. Congenital limb anomalies: frequency and aetiological factors. J Med Gen 1974; 11: 221-33.

${ }^{16}$ Saxén L, Klemetti A, Härö AS. A matched-pair register for studies of selected congenital defects. Am J Epidemiol 1974; 100: 297-306.

${ }^{17}$ Aro T, Heinonen OP, Saxén L. Incidence and secular trends of congenital limb defects in Finland. Int $J$ Epidemiol (in press).

${ }^{18}$ Pitkänen H. Maternity welfare in Finland. In: Public health medical care and the medical profession in Finland. Helsinki: Finnish Medical Association, 1964.

${ }^{19}$ Breslow NE, Day WE, Halvorsen KT, Prentice RL, Saba C. Estimation of multiple relative risk functions in matched case-control studies. Am J Epidemiol 1978 108: 299-307.

${ }^{20}$ Saxén L. Epidemiological strategies of teratoge detection. In: Second international course in industria toxicology. Helsinki: Institute of Occupational Health 1977.

${ }^{21}$ Saxén I. Cleft lip and palate in Finland: parental histories course of pregnancy and selected environmental factors Int J Epidemiol 1974; 3: 263-70.

${ }^{22}$ Nowack E. Die sensible Phase bei de Thalidomid-Embryopathie. Hum Gen 1965; 1: 516-36.

${ }^{23}$ Granroth G. Defects of the central nervous system in Finland. III Diseases and drugs in pregnancy. Early Human Development 1978; 2: 147-62. 